\title{
Assessment of Left Ventricular Filling Dynamics Utilizing Doppler Echocardiography in Acute Coronary Syndrome
}

\author{
Hiroshi Nonogi, M.D., Yoshio Kawase, M.D.,* \\ Shunichi Mryazaki, M.D., Kazuo Haze, M.D., \\ and Katsuhiko HiRAmori, M.D.**
}

\section{SUMMaRY}

To evaluate prolonged diastolic dysfunction in acute coronary syndrome, mitral inflow velocities and left ventricular wall motion were examined with pulsed-wave Doppler and two-dimensional echocardingraphy in 14 patients with successful reperfusion, 8 patients without reperfusion after acute myocardial infarction, and in 10 patients with prolonged angina. Early peak flow velocity and the ratio of early to atrial peak flow velocity increased gradually both after the onset of acute myocardial infarction with reperfusion and cessation of angina pectoris. However, in acute myocardial infarction without reperfusion, early peak flow velocity and the ratio of early to atrial peak flow velocity decreased significantly. Atrial peak flow velocity remained unchanged in acute myocardial infarction with reperfusion, while it was decreased in angina pectoris on the seventh day. Regional systolic dysfunction abated gradually in acute myocardial infarction with successful reperfusion, and regional systolic dysfunction was not detected in angina pectoris on the third day. Thus, abnormalities in left ventricular early diastolic filling persisted for several days after reperfusion in acute myocardial infarction or cessation of angina. Left ventricular late filling remained unchanged in acute myocardial infarction, whereas, in angina pectoris, a compensatory increase in late filling diminished gradually with an increase in carly filling. Thesc results indicate that thcre are different types of recovery process after diastolic filling abnormalities in patients with acute coronary syndrome. (Jpn Heart J 35: 163-173, 1994)

Key words: Doppler echocardiography Ischemia Filling Reperfusion Stunned myocardium

$\mathrm{R}^{\mathrm{R}}$ ECENTLY, several investigators have shown prolonged left ventricular systolic dysfunction after transient myocardial ischemia ("stunned myocardium"). "It is well known that acute myocardial infarction leads to an impairment

\footnotetext{
From the Division of Cardiology, Department of Internal Medicine, National Cardiovascular Center, Suita, Japan.

Address for correspondence: Hiroshi Nonogi, M.D., Division of Cardiology, Department of Internal Medicine, National Cardiovascular Center, 5-7-1, Fujishirodai, Suita 565, Japan.

Present address: *Izumi Municipal Hospital, 4 10-10 Fuchu-cho, Izumi 594, Japan, and **Iwate Medical College, 19-1 Uchimaru, Morioka 020, Japan.

Received for publication October 22, 1993.

Accepted January 10, 1994.
} 
in left ventricular global systolic function with regional wall motion abnormalities and abnormal diastolic filling patterns in the chronic phase. ${ }^{2)}$ During acute ischemia, left ventricular systolic dysfunction is accompanied by diastolic dysfunction which sometimes precedes systolic dysfunction. ${ }^{3,4)}$ However, little is known about the nature and extent of left ventricular diastolic dysfunction during myocardial stunning. ${ }^{5}$ '

The recent development of Doppler echocardiography has allowed the noninvasive measurement of left ventricular diastolic filling, which correlates well with other measures of left ventricular filling. ${ }^{6-9)}$ Therefore, in this study, we studied serial changes in diastolic filling after myocardial ischemia with pulsedwave Doppler analysis of mitral inflow in patients with acute coronary syndrome to assess whether or not there is a different recovery process in diastolic filling abnormalities between acute myocardial infarction and unstable angina.

\section{Methods}

Patients: A total of 32 patients were included in the present study (25 men, 7 women; Table I). Twenty-two patients had acute myocardial infarction as determined by a typical history, standard electrocardiographic changes and an increase in cardiac enzymes. Fourteen of these patients (anterior, 9; inferior, 5) were successfully reperfused within 6 hours after the onset: 5 patients with intracoronary thrombolysis, 2 patients with direct coronary angioplasty, 6 patients with intracoronary thrombolysis followed by angioplasty and 1 patient with spontaneous recanalization. In 8 of the 22 patients with acute myocardial infarction (anterior, 5; inferior, 3), reperfusion therapy was unsuccessful in 2 patients and not performed in 6 patients, who were admitted more than 6 hours

Table I. Demographic and Clinical Parameters

\begin{tabular}{|c|c|c|c|c|}
\hline & & $\begin{array}{l}\text { AMI with reperfusion } \\
\qquad(\mathrm{n}=14)\end{array}$ & $\begin{array}{l}\text { AMI without reperfusion } \\
\qquad(\mathrm{n}=8)\end{array}$ & $\begin{array}{l}\text { Unstable Angina } \\
\qquad(\mathrm{n}=10)\end{array}$ \\
\hline Age & & $57 \pm 8$ & $66 \pm 8$ & $62 \pm 11$ \\
\hline Males & & $11(79 \%)$ & $6(75 \%)$ & $8(80 \%)$ \\
\hline \multicolumn{5}{|c|}{ Extent of CAD } \\
\hline & 0 vessel & $1(7 \%)$ & $0(0 \%)$ & $0(0 \%)$ \\
\hline & I vessel & $5(36 \%)$ & $2(25 \%)$ & $5(50 \%)$ \\
\hline & 2 vessels & $6(43 \%)$ & $3(38 \%)$ & $3(30 \%)$ \\
\hline & 3 vessels & $2(14 \%)$ & $1(12 \%)$ & $2(20 \%)$ \\
\hline \multirow{2}{*}{\multicolumn{2}{|c|}{ Previous MI }} & $0(0 \%)$ & $2(25 \%)$ & $0(0 \%)$ \\
\hline & & 2 & 0 & 3 \\
\hline \multicolumn{2}{|c|}{ Peak CPK (IU/L) } & $3677 \pm 2973$ & $2158 \pm 1824$ & - \\
\hline \multicolumn{2}{|c|}{ Time to peak CPK (hrs) } & $14 \pm 5$ & $2 l \pm 6 *$ & - \\
\hline
\end{tabular}

$\mathrm{AMI}=$ acute myocardial infarction; $\mathrm{CAD}=$ coronary artery disease; $\mathrm{CPK}=$ creatine phosphokinase; ${ }^{*} \mathrm{p}<0.05 \mathrm{vs}$ AMI with reperfusion. 
after the onset of acute myocardial infarction. None of the 22 patients had postinfarction angina or silent myocardial ischemia. Another 10 patients had prolonged angina which persisted more than 15 minutes at rest and were treated with oral anti-anginal medications or intravenous nitroglycerine in the coronary care unit. After angina was controlled, neither angina nor ischemic electrocardiographic changes were observed at all. Patients with mitral regurgitation, a poor recording of Doppler and two-dimensional echocardiograms, tachycardia of more than 90 beats per minute, pulmonary edema or cardiogenic shock were excluded from the study. Patients treated with intraaortic balloon pumping were also excluded.

Study protocol: Two-dimensional and Doppler echocardiographic studies were performed with an ultrasound system (Toshiba SSH-65A) with a $2.5 \mathrm{MHz}$ transducer for both cardiac imaging and determination of flow velocities. Echocardiographic examinations were performed within 24 hours of admission in patients with acute myocardial infarction, and were repeated on the third and seventh days after the onset of acute myocardial infarction. In patients with prolonged angina, the echocardiographic examinations were performed on the first, third and seventh days after the last angina.

Two-dimensional parasternal long-axis and short-axis images were recorded on videotape. A wall motion score index was derived in each patient. ${ }^{10)}$ Briefly, the left ventricle was divided into 9 segments and segmental wall motion was graded in each segment as normal (score, 1); hypokinetic, marked reduction of endocardial motion (score, 2); akinetic, virtual absence of inward motion (score, 3); or dyskinetic, paradoxic wall motion away from the center of the left ventricle in systole (score, 4 ). ${ }^{11}$

Doppler transmitral inflow velocities were recorded in the pulsed-wave mode by positioning the sample volume at the mitral annulus level in the apical four chamber view during an expiratory pause. ${ }^{12)}$ All Doppler recordings with a single lead electrocardiogram were recorded on a strip chart recorder at a paper speed of $100 \mathrm{~mm} / \mathrm{second}$. The pulsed Doppler study was performed in a supine position or the left lateral recumbent position and the transducer was placed at the point of maximal impulse to obtain an apical 4-chamber view of the heart. The sample volume was placed at the level of the mitral valve annulus with the cursor line oriented as parallel as possible to mitral flow. ${ }^{23}$ Flow velocity was not corrected for the angle of the echocardiographic beam. The Doppler velocity curve of five consecutive cardiac cycles was digitized through the darkest gray scale with an electronic digitizer (KD4600, Graphtech, Tokyo), and the variables obtained were averaged. Early peak flow velocity and atrial peak flow velocity as well as the ratio of early to atrial peak flow velocity were determined. During the study period, the same medications were continued in the same patients. All 
patients were examined in the same position, with the same apical window and at almost the same time to reduce the variability of measurements on the day and in serial examinations by the same observer (K.Y.). The echocardiography videotapes were analyzed by two independent observers. When there was disagreement concerning the results, a third observer reviewed the study, and the majority judgment was binding in all cases.

Selective coronary arteriography was performed with the Judkins technique in 30 of 32 patients. Multiple views of each coronary artery were obtained, including craniocaudal views. All angiograms were interpreted by more than two experienced angiographers. Significant coronary stenosis was defined as $70 \%$ or greater reduction in the luminal diameter. The infarct-related artery was identified from the electrocardiogram and the location of wall motion abnormalities by ventriculography. Angiographic reperfusion of the infarct-related artery in the present study was defined as grade 2 or 3 as reported in Thrombolysis in Myocardial Infarction trial. ${ }^{13}$ )

Statistical analysis: Values are expressed as mean values \pm 1 standard deviation in the tables and as mean values \pm 1 standard error in Figure 3. Analysis of variance was used to compare variables in the patient group studied from the first to the seventh day, followed by the Newman-Keul multiple comparison test. A probability value of $\mathrm{p}<0.05$ was considered significant.

\section{Results}

The patients' characteristics are shown in Table I. There were no significant differences among the three groups with regard to age, sex, extent of coronary artery disease or previous myocardial infarction. Peak creatine phosphokinase values were higher in the reperfusion group than in the nonreperfusion group, but not significantly. The time to peak creatine phosphokinase value was significantly shorter in acute myocardial infarction with reperfusion than in that without reperfusion.

Echocardiographic parameters are shown in Table II. Representative serial changes in transmitral flow in myocardial infarction with reperfusion are shown in Figure 1 and in unstable angina in Figure 2. Summarized data for all patients are shown in Figure 3.

General hemodynamics: Systolic and diastolic blood pressures and heart rate remained unchanged during the studies in all 3 groups. On the first day, the wall motion score was not different between the acute myocardial infarction with reperfusion and that without reperfusion, whereas in unstable angina, the wall motion score was almost normal.

Diastolic function on the first day: Early peak flow velocity and the ratio of 
Table II. Serial Changes in Echocardiographic Parameters of 32 patients

\begin{tabular}{l|lll|lll|lll}
\hline & \multicolumn{3}{|c|}{ AMI with reperfusion } & \multicolumn{2}{c|}{ AMI without reperfusion } & \multicolumn{3}{c}{ Unstable AP } \\
\cline { 2 - 10 } & 1 st & 3 rd & 7 th & 1 st & 3 rd & 7 th & 1 st & 3 rd & 7 th \\
\hline sBP (mmHg) & 119 & 117 & 115 & 114 & 123 & 124 & 128 & 129 & 124 \\
& \pm 8 & \pm 15 & \pm 12 & \pm 14 & \pm 16 & \pm 12 & \pm 9 & \pm 14 & \pm 9 \\
$\mathrm{dBP}(\mathrm{mmHg})$ & 73 & 70 & 70 & 68 & 71 & 72 & 74 & 73 & 69 \\
& \pm 11 & \pm 7 & \pm 7 & \pm 8 & \pm 6 & \pm 4 & \pm 8 & \pm 10 & \pm 5 \\
$\mathrm{HR}(\mathrm{bpm})$ & 74 & 73 & 70 & 68 & 76 & 69 & 69 & 64 & 62 \\
& \pm 9 & \pm 8 & \pm 10 & \pm 10 & \pm 6 & \pm 12 & \pm 10 & \pm 12 & \pm 10 \\
Asynergy & 15.5 & 12.6 & 12.1 & 15.1 & 16.3 & 15.4 & 10.0 & 10.0 & 10.0 \\
score & \pm 2.5 & $\pm 2.5 * *$ & $\pm 1.8^{* *}$ & \pm 4.2 & \pm 4.5 & \pm 4.5 & \pm 2.2 & \pm 2.2 & \pm 2.2 \\
E (cm/sec) & 40 & 48 & 50 & 52 & 40 & 38 & 37 & 42 & 48 \\
& \pm 11 & $\pm 10^{* *}$ & $\pm 10^{* *}$ & \pm 14 & $\pm 8^{*}$ & $\pm 8 * *$ & \pm 8 & \pm 8 & $\pm 11^{* *}$ \\
A (cm/sec) & 57 & 62 & 59 & 62 & 71 & 63 & 64 & 58 & 57 \\
& \pm 11 & $\pm 14^{*}$ & \pm 12 & \pm 14 & \pm 9 & \pm 19 & \pm 8 & \pm 6 & $\pm 8^{*}$ \\
E/A & 0.73 & 0.79 & 0.87 & 0.90 & 0.57 & 0.61 & 0.59 & 0.73 & 0.85 \\
& \pm 0.24 & \pm 0.22 & $\pm 0.20^{* *}$ & \pm 0.33 & $\pm 0.16^{* *}$ & \pm 0.21 & \pm 0.11 & $\pm 0.14^{* *} \pm 0.16^{* *}$ \\
\hline
\end{tabular}

$\mathrm{AMI}=$ acute myocardial infarction; $\mathrm{UAP}=$ unstable angina; $\mathrm{sBP}=$ systolic blood pressure; $\mathrm{dBP}=$ diastolic blood pressure; $\mathrm{HR}=$ heart rate; Asynergy score=wall motion score; $\mathrm{E}$-early peak flow velocity; $\mathrm{A}=$ atrial peak flow velocity; $\mathrm{E} / \mathrm{A}=$ the ratio of $\mathrm{E}$ to $\mathrm{A} ;{ }^{* *} \mathrm{p}<0.01,{ }^{*} \mathrm{p}<0.05$ (1 st day vs $3 \mathrm{rd}$ and 7 th day). Meantstandard deviation.

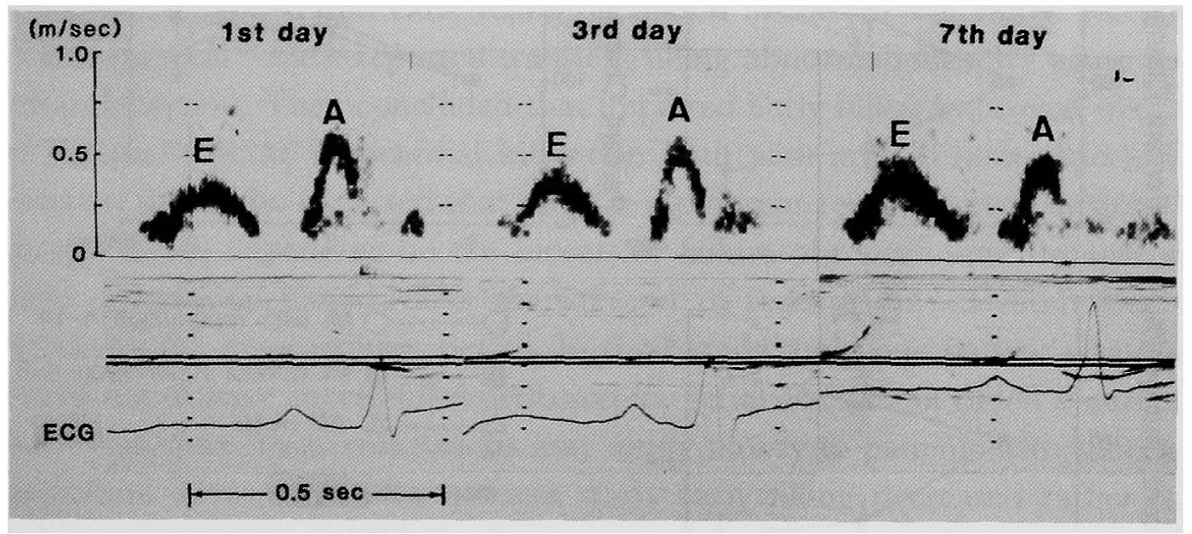

Figure 1. Original records showing serial pulsed Doppler tracings 1,3 and 7 days after the onset of acute myocardial infarction with successful reperfusion of left anterior descending artery. $\mathrm{A}=$ =atrial transmitral flow velocity; $\mathrm{E}=$ =early transmitral flow velocity; ECG=electrocardiogram.

early to atrial peak flow velocity were significantly higher in acute myocardial infarction without reperfusion than in acute myocardial infarction with reperfusion and in unstable angina $(p<0.05)$. Atrial peak flow velocity did not differ significantly among the three groups.

Acute myocardial infarction with reperfusion: An increase in early peak flow velocity was observed 3 days after reperfusion and the ratio of early to atrial peak flow velocity was increased significantly on the seventh day. Atrial peak flow 


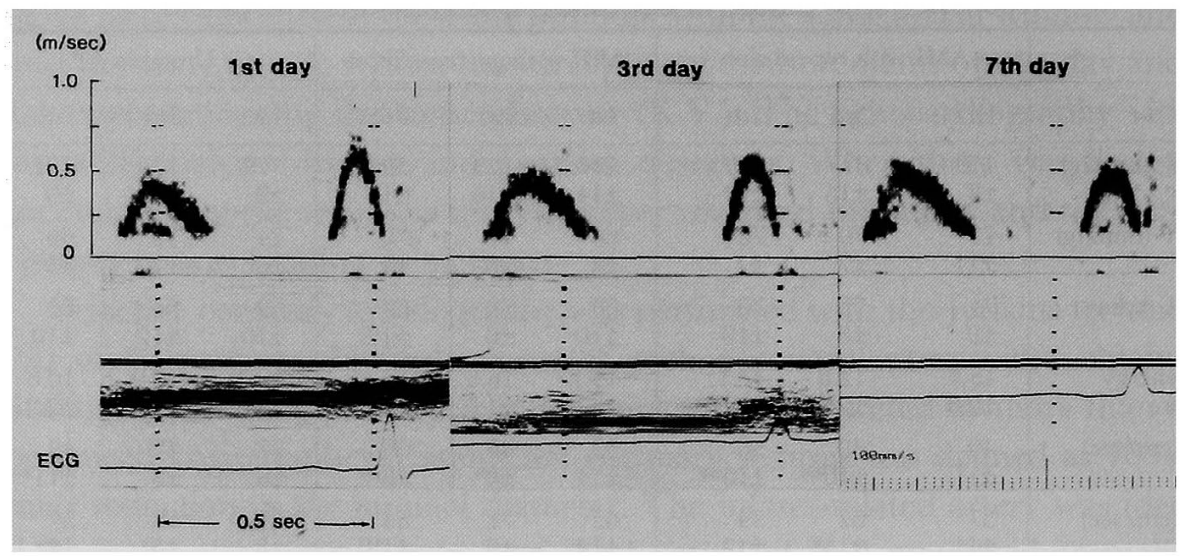

Figure 2. Original records showing serial pulsed Doppler tracings 1,3 and 7 days after remission of angina.
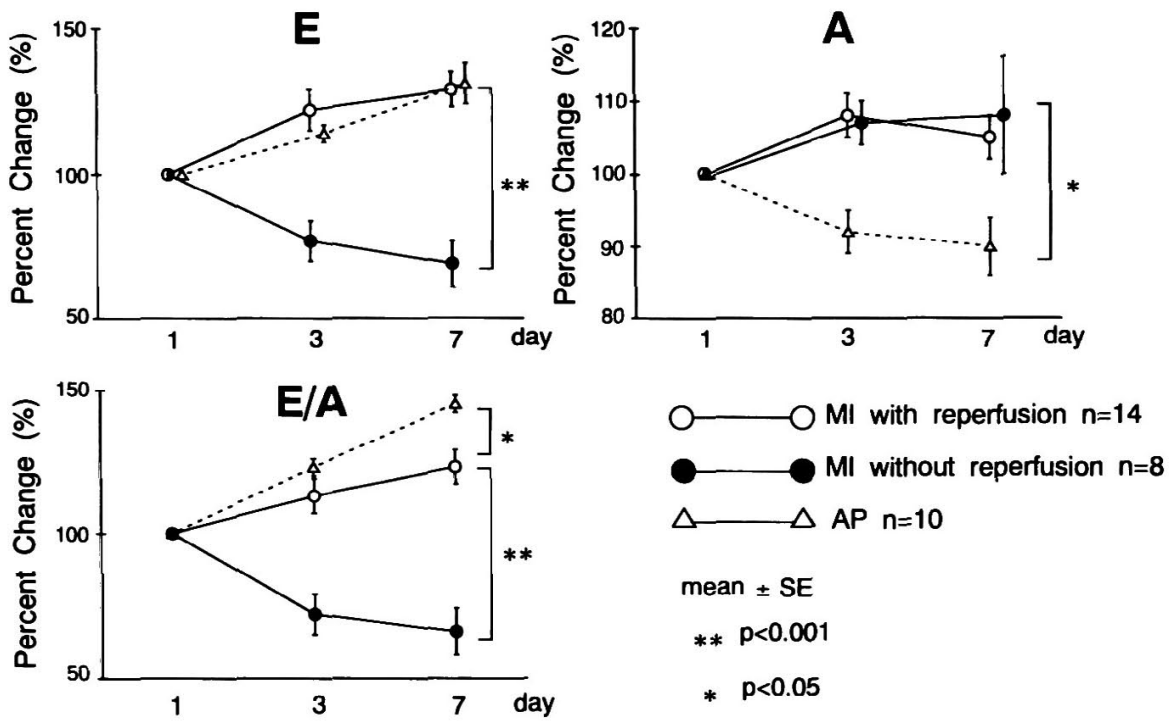

Figure 3. Averaged percent changes 1,3 and 7 days after myocardial infarction (MI) with successful reperfusion (open circles), without reperfusion (closed circles) and unstable angina (triangles). $\quad E=$ =arly peak flow velocity; $A=$ atrial peak flow velocity; $\mathrm{E} / \mathrm{A}=$ the ratio of $\mathrm{E}$ to $\mathrm{A} ; \mathrm{SE}=$ standard error.

velocity remained unchanged. The wall motion score had improved significantly by the third day.

Acute myocadial infarction without reperfusion: Early peak flow velocity and the ratio of early to atrial peak flow velocity decreased significantly on the third and seventh days. Atrial peak flow velocity remained unchanged. Wall 
motion score remained unchanged during the study.

Unstable angina: Early peak flow velocity and the ratio of early to atrial peak flow velocity were significantly increased on the third and seventh days, whereas, atrial peak flow velocity was significantly decreased. The wall motion score was almost normal and remained unchanged after the last episode.

\section{Discussion}

This study demonstrated prolonged diastolic dysfunction both after successful reperfusion in acute myocardial infarction and after cessation of prolonged anginal attacks. The recovery process after diastolic filling abnormalities was quite different depending on the presence or absence of myocardial infarction and success or failure of reperfusion.

Diastolic filling in acute myocardial infarction: After successful reperfusion in patients with acute myocardial infarction, the decreased early filling of the left ventricle improved gradually along with improvement from left ventricular asynergy within the first week. Without reperfusion, regional systolic dysfunction remained unchanged and early filling decreased in the present study. Recently, Williamson et $\mathrm{al}^{(4)}$ have reported transient filling abnormalities after acute myocardial infarction. They concluded that impaired early filling improved over the first 7 days in acute myocardial infarction both with and without reperfusion. However, it may be expected that there is a different recovery process from the ischemic insult, depending on the success or failure of reperfusion, because successful reperfusion would affect the amount of residual myocardium and the function of the myocardium, per se. In their study, only one patient was documented not to have successful reperfusion for serial assessment of left ventricular filling. Therefore, their conclusions may apply mostly to patients with successful reperfusion. However, in the present study early filling decreased rather than improved during the first week after myocardial infarction without reperfusion.

Myocardial infarction without reperfusion: Many factors seem to be involved in the regulation of left ventricular filling dynamics in myocardial infarction. ${ }^{15,16)}$ One of the most important mechanisms which affect early diastolic left ventricular pressure is elastic recoil of the left ventricle created by systolic contraction. ${ }^{17)}$ Because the magnitude of elastic recoil is inversely related to endsystolic volume, elastic recoil is considered to remain decreased due to the lack of improvement in systolic function in acute myocardial infarction without reperfusion. Another mechanism is related to left ventricular relaxation. Influences of relaxation on early diastolic filling have been studied extensively in animal models and in human subjects. ${ }^{15,16,18,19)}$ In myocardial infarction without reperfusion, left ventricular relaxation is prolonged due to both asynchrony and 
loss of myocardial tension which are caused by myocardial necrosis. In myocardial infarction without reperfusion, early peak flow velocity was higher on the first day, probably because of an increase in preload. The mechanisms of the decreased early peak flow velocity 1 week after myocardial infarction without reperfusion could be ascribed to either a reduced preload or impaired relaxation due to a possible increase in asynchrony.

Myocardial infarction with reperfusion: After successful reperfusion, recovery from ischemia in the infarct region is expected to enhance relaxation due to an improvement in shortening and a decrease in asynchrony. Thus, in the reperfusion group, improvement from regional systolic dysfunction resulted in enhancement of elastic recoil and relaxation that would decrease the left ventricular early diastolic pressure with an improvement in early filling. However, atrial peak flow velocity remained unchanged in the present study. The improvement of regional systolic function might induce the increases in stroke volume and total filling volume which result in an increase in early filling without the changes in late diastolic filling. Tilton et $\mathrm{al}^{20)}$ examined left ventricular segmental relaxation in dogs undergoing 2 or 4 hours of coronary occlusion followed by reperfusion. They found that the recovery of diastolic function occurred in the first week after acute myocardial infarction. The findings of this experimental study support our clinical observation of an improvement from regional systolic dysfunction and of better diastolic early filling during the first week after acute myocardial infarction with reperfusion. These findings suggest stunning of both systolic and diastolic properties. ${ }^{5,21)}$

Diastolic filling in unstable angina: During remission after prolonged anginal attacks, regional systolic dysfunction was not detected in two-dimensional echocardiography, but, early filling was still impaired with a compensatory increase in late filling. Thereafter, early filling improved gradually with a decrease in late filling. In previous studies, both systolic and diastolic dysfunction were observed during and immediately after acute ischemia. ${ }^{4,22)}$ However, little is known about the process of recovery of diastolic function after myocardial ischemia. Iliceto et $\mathrm{al}^{233}$ reported that a significant rearrangement of left ventricular filling occurred after pacing-induced ischemia in patients with stable angina. Immediately after rapid atrial pacing, early filling decreased with a compensatory increase in atrial filling, and the filling alterations gradually returned to preischemic values without changes in systolic function. ${ }^{23)}$ The duration of diastolic dysfunction was shorter in their study than in the present study. This could be ascribed to the difference in severity of the preceding ischemia, because their pacing-induced ischemia for stable angina would be milder than that in the prolonged angina in the present study.

The mechanisms for the observed decrease in early peak flow velocity were 
not confirmed directly in our study because serial measurements of left ventricular pressure could not be obtained. However, because early diastolic filling is influenced by the pressure gradient between the left atrium and the left ventricle ${ }^{16)}$ the observed decrease in early peak flow velocity could be ascribed to an increase in left ventricular diastolic pressure and/or a decrease in left atrial pressure which may be caused by changes in elastic recoil and/or left ventricular relaxation. Immediately after severe ischemia, transient systolic and diastolic filling abnormalities are observed ${ }^{4,22)}$ In this situation with systolic dysfunction, it is speculated that serial changes in diastolic filling abnormalities might be similar to those in myocardial infarction with successful reperfusion. However, systolic function was well reserved after the remission from angina in the present study. Therefore, it seems unlikely that the changes can be explained solely by decreased elastic recoil. Pasipoularides et $\mathrm{al}^{243}$ reported that incomplete relaxation induces an increasc in relaxation pressure in the early diastolic phase which results in an elevated early diastolic pressure. Murakami et $\mathrm{al}^{25)}$ showed that both left ventricular relaxation and early diastolic filling were improved after treatment with diltiazem despite a decrease in myocardial contractility. In a recent experimental study in our institute, early filling and relaxation were impaired during pacing-induced stunned myocardium. ${ }^{26)}$ These data suggest that impaired relaxation is a major mechanism in the decrease of early filling after remission from angina in hearts with normal systolic function. Because of maintained systolic function which means little change in total filling volume, a compensatory increase in late filling diminished gradually with an increase in early filling to keep a constant total filling volume.

Limitation of study: Several studies have shown the effect of preload on filling dynamics. ${ }^{27-29)}$ In general, it can be expected that a higher filling pressure occurs during the early phase of acute myocardial infarction which augments early filling, In patients without reperfusion, a decrease in early filling might be dependent on the preload change during the first week after acute myocardial infarction. In the present study, patients with pulmonary edema were excluded because they have tachycardia and summation of early and late filling patterns Therefore, the present study did not show changes of filling dynamics in patients with severely reduced left ventricular function. An increase in left atrial pressure with left ventricular dysfunction induces "pseudo-normalization" of mitral flow velocity pattern. ${ }^{29)}$ Therefore, in patients with "pseudo-normalization" pattern after successful reperfusion, serial changes in filling abnormalities were different from those in the present study. This may be a limitation of this study. In the present study, the observed findings are limited to a few cases in acute coronary syndrome without congestive heart failure. Therefore, further study is necessary to analyze the serial changes of pseudonormalized filling pattern after successful 
reperfusion.

In conclusion, abnormalities of left ventricular early diastolic filling induced by severe ischemia persisted for several days and then improved within one week in patients with acute myocardial infarction treated with successful reperfusion and in those with prolonged angina. However, in patients with acute myocardial infarction without reperfusion, decreased left ventricular filling dynamics persisted for more than a week. Left ventricular late filling remained unchanged in patients with myocardial infarction, although a compensatory increase in late filling gradually diminished with an increase in early filling in patients with angina pectoris, suggesting redistribution of the diastolic filling fraction. Thus, there are different types of diastolic filling abnormalities in patients with acute coronary syndrome.

\section{ACKNowLedgment}

The authors would like to thank Dr. A.S. Cary, M.D. for reading the manuscript.

\section{REFERENGES}

1. Braunwald E, Kloner RA: The stunned myocardium; prolonged post-ischemic ventricular dysfunction. Circulation 66: 1146, 1982

2. Bonow RO, Bacharach SL, Green MV, Kent KM, Rosing DR, Lipson LC, Leon MB, Epstein SE: Impaired left ventricular diastolic flling in patients with coronary artery disease; assessment with radionuclide angiography. Circulation 64: 315, 1981

3. Aroesty JM, McKay RG, Heller GV, Royal HD, Als AV, Grossman W: Simultaneous assessment of left ventricular systolic and diastolic dysfunction during pacing-induced ischemia. Circulation 71: 889, 1985

4. Nonogi $\mathrm{H}$, Sasayama S, Miyazaki S, Kawai C: An analysis of regional left ventricular function utilizing pressure-length relationship in patients with coronary artery disease. Jpn Circ J 51: 83, 1987

5. Przyklenk K, Pate B, Kloner RA: Diastolic abnormalities of postischemic "Stunned" myocardium. Am J Cardiol 15: 1211, 1987

6. Ferguson IIIJJ, Manning WJ, Come PC: Pulsed Doppler echocardiographic determination of the time course of left ventricular filling; validation with cineangiography. Am Heart J 117: 127, 1988

7. Spirito P, Maron BJ, Bonow RO: Noninvasive assessment of left ventricular diastolic function; comparative analysis of Doppler echocardiographic and radionuclide angiographic techniques. $\mathrm{J}$ Am Coll Cardiol 7: 518,1986

8. Friedman BJ, Drinkovic N, Miles H, Shin WJ, Mazzolein A, De Maria AN: Assessment of left ventricular diastolic function; comparison of Doppler echocardiographic and gated blood pool scintigraphy. J Am Coll Cardiol 8: 1348, 1986

9. Rokey R, Kuo LC, Zoghbi WA, Limacher MC, Quinones MA: Determination of parameters of left ventricular diastolic filling with pulsed Doppler echocardiography; comparison with cineangiography. Circulation 71: 543,1985

10. Heger JJ, Weyman AE, Wann LS, Dillon JC, Feigenbaum H: Cross-sectional echocardiography in acute myocardial infarction; detection and localization of regional left ventricular asynergy. Circulation 60: 531,1979

11. Bolognese L, Sarasso G, Bongo AS, Rossi L, Aralda D, Piccinino C, Rossi P: Dipyridamole echocardiography test; a new tool for detecting jeopardized myocardium after thrombolytic therapy. 
Circulation 84: 1100, 1991

12. Miyatake K, Okamoto M, Kinoshita N, Nakasone I, Sakakibara H, Nimura Y: Augmentation of atrial contribution to left ventricular inflow with aging as assessed by intracardiac Doppler flowmetry. Am I Cardiol 53: 586, 1984

13. Chesebro JH, Kuatterud G, Roberts R, Borer J, Cohen LS, Dalen J, Dodge HT, Francis CK, Hillis D, Ludbrook P, Markis JE, Mueller H, Passamani ER, Power ER, Rao AK, Robertson T, Ross A, Ryan TJ, Sobel BE, Willerson J, Williams DO, Zaret BL, Braunwald E: Thrombolysis in myocardial infarction (TIMI) trial, phase I; a comparison between intraverous tissue plasminogen activator and intravenous streptokinase. Circulation 76: 142, 1987

14. Williamson BD, Lim MJ, Buda AJ; Transient left ventricular filling abnormalities (diastolic stunning) after acute myocardial infarction. Am J Cardiol 66: 897, 1990

15. Choong CY, Abascal VM, Thomas JD, Guerrero JL, McGlew S, Weyman AE: Combined infuence of ventricular loading and relaxation on the transmitral flow velocity profle in dogs measured by Doppler echocardiography. Circulation 78: 672, 1988

16. Ishida Y, Meisner JS, Tsujioka K, Gallo JI, Yoran C, Frater RWM, Yellin EL: Left ventricular filling dynamics; infuence of left ventricular relaxation and left atrial pressure. Circulation 74: 187, 1986

17. Sonnenblick EH: The structural basis and importance of restoring forces and elastic recoil for the filling of the heart. Eur Heart J 1 (Suppl A): 107, 1980

18. Carroll JD, Widmer $\mathrm{R}$, Hess $\mathrm{OM}$, Hirzel HO, Krayenbuehl HP: Left ventricular isovolumic pressure decay and diastolic mechanics after postextrasystolic potentiation and during exercise. Am J Cardiol $\mathbf{5 1}$ : 583,1983

19. Carroll JD, Hess OM, Hirzel HO, Krayenbuehl HP: Dynamics of left ventricular filling at rest and during exercise. Circulation 68: 59, 1983

20. Tilton GD, Bush LR, Apprill PG, Buja LM, Willerson JT: Effect of diltiazem and propranolol on left ventricular segmental relaxation during temporary coronary arterial occlusion and one month reperfusion in conscious dogs. Girculation 71: 165, 1985

21. Charlat ML, O'Neill PG, Hartley CJ, Roberts R, Bolli R: Prolonged abnormalities of left ventricular diastolic wall thinning in the "stunned" myocardium in conscious dogs; time course and relation to systolic function. J Am Coll Cardiol 13: 185, 1989

22. Nakamura Y, Sasayama S, Nonogi H, Miyazaki S, Fujita M, Kihara Y, Konishi T, Kawai C: Effects of pacing-induced ischemia on early left ventricular filling and regional myocardial dynamics and their modification by nifedipine. Circulation 76: 1232, 1987

23. Iiceto S, Amico A, Marangelli V, D'Ambrosio G, Rizzon P: Doppler echocardiographic evaluation of the effect of atrial pacing-induced ischemia on left ventricular filling in patients with coronary artery disease. J Am Coll Cardiol 11: 953, 1988

24. Pasipoularides A, Mirsky I, Hess OM, Grimm J, Krayenbuehl HP: Myocardial relaxation and passive diastolic properties in man. Circulation 74: 991,1986

25. Murakami $T$, Hess OM, Krayenbueh HP: Left ventricular function before and after Diltiazem in patients with coronary artery disease. J Am Coll Cardiol 5: 723, 1985

26. Miyazaki S, Goto Y, Nonogi H, Kawase Y, Haze K, Hiramori K: Impaired early and intact late diastolic function in stumed myocardium induced by demand ischemia. Am J Physiol 264: H739, 1993

27. Courtois M, Vered Z, Barzilai B, Ricciotii NA, Perez JE, Ludbrook PA: The transmitral pressure-flow relation; effect of abrupt preload reduction. Circulation 78; 1459, 1988

28. Stoddard MF, Person AC, Kern MJ, Ratcliff J, Mrosek DG, Labovitz AJ: Left ventricular diastolic function; comparison of pulsed Doppler echocardiographic and hemodynamic indexes in subjects with and without coronary artery disease. J Am Coll Cardiol 13: 327, 1989

29. Appleton CP, Hatle LK, Popp RL: Relation of transmitral flow velocity patterns to left ventricular diastolic function; new insights from a combined hemodynamic and Doppler echocardiographic study. J Am Coll Cardiol 12: 426, 1988 